\title{
Formononetin attenuates monocrotaline-induced pulmonary arterial hypertension via inhibiting pulmonary vascular remodeling in rats
}

\author{
CHANGHONG CAI $^{1}$, YIJIA XIANG ${ }^{1}$, YONGHUI WU ${ }^{1}$, NING ZHU $^{2}$, HUAN ZHAO $^{1}$, \\ JIAN XU ${ }^{1}$, WENSHENG LIN ${ }^{1}$ and CHUNLAI ZENG ${ }^{1}$
}

\begin{abstract}
${ }^{1}$ Department of Cardiology, The Fifth Affiliated Hospital of Wenzhou Medical University, Lishui Central Hospital, Lishui, Zhejiang 323000; ${ }^{2}$ Department of Cardiology, The Third Clinical College of Wenzhou Medical University, Wenzhou People's Hospital, Wenzhou, Zhejiang 325000, P.R. China
\end{abstract}

Received September 21, 2018; Accepted March 15, 2019

DOI: $10.3892 / \mathrm{mmr} .2019 .10781$

\begin{abstract}
Pulmonary arterial hypertension (PAH) is a life-threatening disease induced by the excessive proliferation and reduced apoptosis of pulmonary artery smooth muscle cells (PASMCs). Formononetin (FMN) is a natural isoflavone with numerous cardioprotective properties, which can inhibit the proliferation and induce the apoptosis of tumor cells; however, whether FMN has a therapeutic effect on PAH remains unclear. In the present study, PAH was induced in rats with monocrotaline (MCT, $60 \mathrm{mg} / \mathrm{kg}$ ); rats were then administered FMN (10,30 or $60 \mathrm{mg} / \mathrm{kg} / \mathrm{day})$. At the end of the experiment, hemodynamic changes, right ventricular hypertrophy and lung morphological characteristics were evaluated. $\alpha$-smooth muscle actin ( $\alpha$-SMA), proliferating cell nuclear antigen (PCNA), and TUNEL were detected by immunohistochemical staining. The expression of PCNA, Bcl-2-associated X protein (Bax), Bcl-2 and, cleaved caspase-3, and activation of AKT and ERK were examined by western blot analysis. The results demonstrated that FMN significantly ameliorated the right ventricular systolic pressure, right ventricular hypertrophy, and pulmonary vascular remodeling induced by MCT. FMN also attenuated MCT-induced increased expression of $\alpha$-SMA and PCNA. The ratio of Bax/Bcl-2 and cleaved caspase-3 expression increased in rat lung tissue in response to FMN treatment. Furthermore, reduced phosphorylation of AKT and ERK was also observed in FMN-treated rats. Therefore, FMN may provide protection against MCT-induced PAH by
\end{abstract}

Correspondence to: Dr Chunlai Zeng, Department of Cardiology, The Fifth Affiliated Hospital of Wenzhou Medical University, Lishui Central Hospital, 289 Kuocang Road, Lishui, Zhejiang 323000, P.R. China

Email: zengchunlai@aliyun.com

Key words: formononetin, pulmonary arterial hypertension, pulmonary vascular remodeling, proliferation, apoptosis, monocrotaline preventing pulmonary vascular remodeling, potentially by suppressing the PI3K/AKT and ERK pathways in rats.

\section{Introduction}

Pulmonary arterial hypertension (PAH) is a multifactorial and devastating cardiopulmonary disorder characterized by the progressive sustained increase in pulmonary arterial pressure, which leads to right ventricular hypertrophy and eventually heart failure (1-4). Pulmonary vascular remodeling caused by excessive proliferation and apoptosis resistance of vascular smooth muscle cells is a prominent feature of PAH (5-7). The majority of existing medications (endothelin antagonists, prostacyclins and phosphodiesterase type 5 inhibitors) alleviate $\mathrm{PAH}$ by mainly dilating partially occluded vessels, rather than inhibiting proliferation and promoting the apoptosis of the pulmonary artery smooth muscle cells (PASMCs) $(8,9)$. Thus, the mortality of PAH is high, $\leq 15 \%$ a year, as are the costs of clinical patient care $(5,8,10)$. Furthermore, numerous studies have validated that the phosphoinositide 3-kinase (PI3K)/protein kinase $\mathrm{B}$ (AKT) and extracellular signal-regulated kinase (ERK) pathways are involved in the survival and apoptosis of PASMCs in PAH (11-13), and are therefore considered therapeutic targets for the treatment of PAH.

In recent years, traditional Chinese medicine has received increasing attention due to its potential advantages, including abundant natural resources, strong targeting potentials, and reduced costs (14). Formononetin (FMN) is an active isoflavone extracted from the traditional Chinese herb Trifolium pratense L. $(15,16)$, which has been widely used to treat cardiovascular diseases $(17,18)$. FMN also exhibits strong antitumor activity in human prostate cancer cells and nasopharyngeal carcinoma cells $(19,20)$. This novel compound has been reported to inhibit proliferation and induce the apoptosis of tumor cells by increasing the B-cell lymphoma 2 (Bcl-2)-associated $\mathrm{X}$ protein (Bax)/Bcl-2 ratio and activating caspases (21-23). In addition, the induction of FMN-mediated apoptosis and the inhibition of proliferation are associated with the inactivation of AKT and ERK signaling in various cell types $(24,25)$; however, the 
therapeutic effects of FMN on PAH and its possible mechanisms remain unknown.

Based on these previous findings, we proposed that FMN could attenuate PAH by inhibiting pulmonary vascular remodeling. In the present study, we explored the protective effects of FMN on the progression of PAH induced by monocrotaline (MCT). Furthermore, the effects of FMN on the apoptosis and proliferation of PASMCs, and underlying molecular mechanisms in vivo were also investigated.

\section{Materials and methods}

Chemicals and reagents. FMN (purity $>98.0 \%$ ) was purchased from MedChem Express, LLC, (New Jersey, USA). MCT, dimethyl sulfoxide (DMSO), bovine serum albumin (BSA) and anti- $\alpha$-smooth muscle actin ( $\alpha$-SMA) antibody were purchased from Sigma-Aldrich; Merck KGaA (Darmstadt, Germany). FMN was dissolved in DMSO and diluted with olive oil $(45 \mathrm{mg} / \mathrm{ml})$. MCT was dissolved in $1 \mathrm{M}$ HCL neutralized with $1 \mathrm{M} \mathrm{NaOH}$, and diluted with normal saline. Then, the $\mathrm{pH}$ was adjusted to 7.2-7.4. Anti-cleaved caspase-3, anti-GAPDH, anti-phosphorylated-AKT, anti-AKT, anti-P-ERK, anti-ERK, anti-rabbit IgG horseradish peroxidase (HRP)-conjugated and anti-mouse IgG HRP-conjugated antibodies were obtained from Cell Signaling Technology, Inc., Danvers, MA, USA. Anti-Bax, anti-Bcl-2, and anti-proliferating cell nuclear antigen (PCNA) antibodies were purchased from Abcam, Cambridge, UK. An H\&E assay kit was obtained from Beijing Solarbio Science \& Technology Co., Ltd., Beijing, China. BCA Protein and Colorimetric TUNEL Apoptosis assay kits were purchased from Beyotime Institute of Biotechnology.

Animals. Male Sprague-Dawley (7-weeks-old) rats weighing 230-250 g were obtained from the Experimental Animal Center of Zhejiang. The experimental protocol was approved by the Ethics Review of Animal Use Application of The Fifth Affiliated Hospital of Wenzhou Medical University (Wenzhou, China) in accordance with the National Institutes of Health Guidelines For the Care and Use of Experimental Animals (26). All rats were housed in an environmentally controlled room at $20-26^{\circ} \mathrm{C}, 50 \pm 5 \%$ humidity under a $12 \mathrm{~h}$ light/dark cycle, and had free access to food and water. After 1 week of acclimation, 68 rats were randomly divided into five groups: i) The control group $(n=8)$ which received normal saline; ii) the MCT group $(\mathrm{n}=15)$ received MCT at $60 \mathrm{mg} / \mathrm{kg}$; iii) the FMN-low group $(n=15)$ received MCT + FMN at $10 \mathrm{mg} / \mathrm{kg} / \mathrm{day}$; iv) the FMN-medium group $(\mathrm{n}=15)$ received $\mathrm{MCT}+\mathrm{FMN}$ at $30 \mathrm{mg} / \mathrm{kg} /$ day; and v) the FMN-high group $(\mathrm{n}=15)$ received $\mathrm{MCT}+\mathrm{FMN}$ at $60 \mathrm{mg} / \mathrm{kg} / \mathrm{day}$. PAH was induced as described previously (27). MCT was administered from at day 0; after 2 weeks, the rats in each FMN group were intraperitoneally administered with different doses of FMN and maintained daily for 2 weeks.

Humane endpoints were set according to the Organisation for Economic Co-operation and Development Guidance document on the Recognition, Assessment, and Use of Clinical Signs as Humane End points for Experimental Animals Used in Safety Evaluation (https://www.aaalac.org/accreditation/RefResources/RR_HumaneEndpoints.pdf). Specifically, as 1 rat demonstrated a reduction in body temperature, dyspnea, cyanosis, appeared hunched with decreased activity and no response to touch, and abrupt weight loss with a reduction in body weight of $>10 \%$ per day for 2 days, the rat was euthanized. On the 22nd day of the experiment, a rat in the MCT group was humanely sacrificed. On day 24, a rat of the FMN-Low group was euthanized. On day 25, two rats of the MCT group and one rat of the FMN-medium group were sacrificed. On day 26, an FMN-low group rat was sacrificed. On day 27, one rat in the FMN-medium and the FMN-High groups were euthanized. All of the animals that survived were weighed weekly for 4 weeks, and were used for hemodynamic and histological analysis. The animals treated with MCT that succumbed during the experimental procedure due to $\mathrm{PAH}$ or heart failure were used only for survival analysis.

Hemodynamic analysis. After weighing, the rats were anesthetized with sodium pentobarbital $(30 \mathrm{mg} / \mathrm{kg})$ intraperitoneally. Then, the right ventricle (RV) systolic pressure (RVSP) was measured as previously reported (27). Once the hemodynamic data had been collected, animals were sacrificed via an intraperitoneal injection of $150 \mathrm{mg} / \mathrm{kg}$ sodium pentobarbital. The RV was separated and weighed. The left lung tissues were excised for histological and immunohistochemical analyses, while the right lung tissues were stored at $-80^{\circ} \mathrm{C}$ for western blot analysis.

Evaluation of right heart hypertrophy. Following hemodynamic analysis, the hearts were separated, dissected into the $\mathrm{RV}$, left ventricle (LV) and septum (S), and weighed, respectively. The weight ratio of RV to LV plus $S$, and the weight ratio of RV to body weight (BW) were calculated as indexes to reflect RV hypertrophy.

Assessment of pulmonary vascular remodeling. The isolated left lungs were fixed in $4 \%$ paraformaldehyde at room temperature for $48 \mathrm{~h}$, embedded in paraffin and sectioned at 4- $\mu \mathrm{m}$ thickness. H\&E staining was performed on the lung tissue sections according to the manufacturer's protocols, the structural remodeling of pulmonary arteries was analyzed via microscopy. In pulmonary arteries with diameters from 50-150 $\mu \mathrm{m}$, the wall thickness (WT) was measured under a light microscope (Nikon Corporation) at a magnification of $\mathrm{x} 400$. For each artery, the degree of WT was calculated as follows: The ratio of vascular WT\% $=100 \% \times \mathrm{WT} /$ outer diameter and the ratio of the vascular wall area $(\mathrm{WA} \%)=100 \%$ $\mathrm{x}$ transection area of the wall/cross-sectional area. A random selection of five fields of six sections were selected for analysis per each group.

Immunohistochemistry. Lung tissue sections (4- $\mu \mathrm{m})$ were dewaxed, rehydrated and then washed with PBS (pH 7.2-7.4). Following antigen retrieval at $100^{\circ} \mathrm{C}$ and blocking with $5 \%$ $\mathrm{BSA}$ at room temperature for $1 \mathrm{~h}$, the sections were incubated with anti- $\alpha$-SMA antibody (1:500, ab2547) or anti-PCNA antibody (1:200, ab18197) overnight at $4^{\circ} \mathrm{C}$, followed by the anti-rabbit $(1: 50,7074 \mathrm{~S})$ or anti-mouse $(1: 50,7076 \mathrm{~S})$ horseradish peroxidase (HRP)-conjugated secondary antibody. Following washing with PBS, the sections were visualized with 3'3'-diaminobenzidene (DAB) at room temperature for $5 \mathrm{~min}$ and counterstained with hematoxylin at room temperature for 
2 min. Apoptosis was analyzed via a terminal deoxynucleotidyl-transferase-mediated dUTP nick end (TUNEL) assay in accordance with the manufacturer's instructions. DAB was used as the chromogen and counterstained with hematoxylin at room temperature for $2 \mathrm{~min}$. Subsequently, the stained sections were observed with a light microscope (magnification, $\mathrm{x} 400$; Nikon Corporation, Tokyo, Japan). The integrated optical density (IOD) of $\alpha$-SMA in the pulmonary arteriole was d using Image-Pro Plus software (Media Cybernetics, Inc., Rockville, MD, USA), and then the ratio of the IOD to the area of the arteriole was calculated to reflect the expression of $\alpha$-SMA. In addition, the percentages of PCNA-positive and TUNEL-positive PASMCs were evaluated in each group.

Western blotting. Proteins were extracted from the lung tissues using lysis buffer containing protease (Beyotime Institute of Biotechnology, Haimen, China) and phosphatase inhibitors (Cell Signaling Technology, Inc.) under homogenization. The protein concentration was measured with the BCA Protein Assay kit. An equivalent amount $(50 \mu \mathrm{g})$ of protein lysate was separated with $10-12 \%$ SDS-PAGE and transferred to a polyvinylidene difluoride membrane. Subsequently, the membranes were blocked with 5\% BSA at room temperature for $2 \mathrm{~h}$ and incubated with anti-PCNA antibody (1:1,000, cat. no. ab18197), anti-Bax antibody (1:1,000, cat. no. ab32503), anti-Bcl-2 antibody (1:1,000, cat. no. ab59348), anti-Cleaved caspase-3 antibody (1:1,000, cat. no. 9661S), anti-Caspase-3 antibody (1:1,000, cat. no. 9662S), anti-P-ERK antibody (1:1,000, cat. no. 9101S), anti- ERK antibody (1:1,000, cat. no. 9102S), anti-P-AKT antibody (1:1,000, cat. no. 4060S), anti-AKT antibody (1:1,000, cat. no. 9272S), overnight at $4^{\circ} \mathrm{C}$. Then, the membranes were washed with TBST and incubated with anti-rabbit IgG HRP-conjugated antibody (1:1,000, cat. no. $7074 \mathrm{~S})$ or anti-mouse IgG HRP-conjugated antibody $(1: 1,000$, cat. no. $7076 \mathrm{~S})$ at room temperature for $1 \mathrm{~h}$. The protein bands were visualized using enhanced chemiluminescence (EMD Millipore, Billerica, MA, USA). The content of protein was analysis by densitometric quantification using AlphaView software (ProteinSimple, San Jose, CA, USA). GAPDH (1:1,000, cat. no. 5174S) was used as an internal control.

Statistical analysis. The data were presented as the mean \pm standard error of the mean, and analysis was performed with GraphPad Prism 5 software (GraphPad Software, Inc., La Jolla, CA, USA). Differences between groups were analyzed by one-way analysis of variance and a Student-Newman-Keuls post-hoc test for multiple comparisons. Kaplan Meier analysis was used to analyze the survival rate of rats. $\mathrm{P}<0.05$ was considered to indicate a statistically significant difference.

\section{Results}

FMN improves the survival rate of rats with $P A H$. No rats succumbed after 28 days in the control group; however, $50 \%$ rats in the MCT group succumbed. On the contrary, treatment with FMN $(60 \mathrm{mg} / \mathrm{kg})$ increased the survival of rats with PAH $(\mathrm{P}<0.05$; Fig. 1).

FMN alleviates MCT-induced hemodynamic alterations and $R V$ hypertrophy. MCT induced a significant increase in RVSP compared with the control $(\mathrm{P}<0.01$; Fig. $2 \mathrm{~A})$. Additionally,

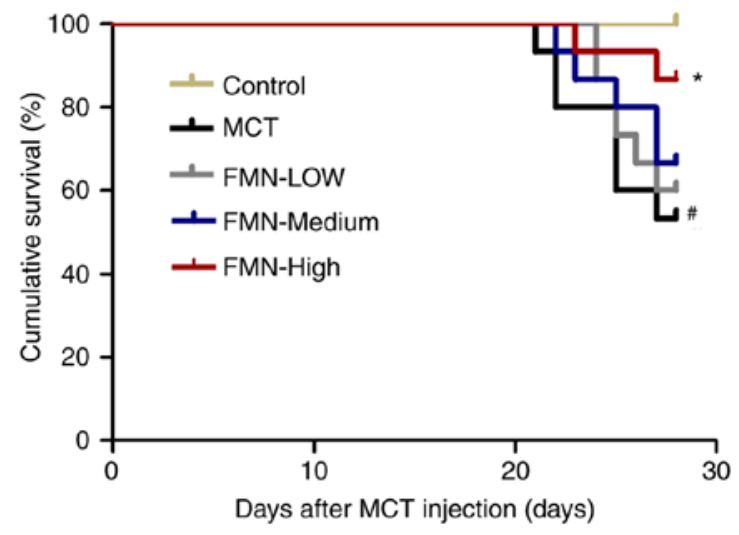

Figure 1. Effects of FMN on the survival of rats. The survival rate was analyzed by the Kaplan-Meier method. ${ }^{~} \mathrm{P}<0.05$ vs. the control group. ${ }^{*} \mathrm{P}<0.05$ vs. the MCT group. $\mathrm{n}=8-15$ per group. FMN, formononetin; MCT, monocrotaline; FMN-Low, 10 mg/kg FMN; FMN-Medium, 30 mg/kg FMN; FMN-High, $60 \mathrm{mg} / \mathrm{kg}$ FMN.

there were no significant differences in RVSP following low- and medium-dose FMN treatments (10 and $30 \mathrm{mg} / \mathrm{kg}$ ) compared with the MCT group; however, high-dose FMN (60 mg/kg) administration significantly decreased the RVSP $(\mathrm{P}<0.01)$. The right ventricular hypertrophy index (RVHI) and $\mathrm{RV} / \mathrm{BW}$ ratio were calculated to evaluate the extent of RV hypertrophy. MCT significantly increased the RVHI compared with the control group, but was significantly reduced following treatment with $60 \mathrm{mg} / \mathrm{kg}$ FMN ( $\mathrm{P}<0.01$; Fig. 2B). In addition, MCT-treated rats exhibited a significantly increased RV/BW ratio than the control group. FMN treatment reduced this ratio in a dose-dependent manner $(\mathrm{P}<0.05$; Fig. $2 \mathrm{C})$.

FMN suppresses MCT-induced pulmonary vascular remodeling. To evaluate pulmonary vascular remodeling, we measured the WT in small pulmonary arteries (external diameters of 50-150 $\mu \mathrm{m}$ ) by H\&E staining. After MCT treatment, the indices of the WT of pulmonary arterioles and the WA were significantly increased compared with the control $(\mathrm{P}<0.01)$. FMN (30 and $60 \mathrm{mg} / \mathrm{kg}$ ) treatment significantly inhibited these pathological alternations in the lungs compared with the MCT group ( $\mathrm{P}<0.05$; Fig. 3).

FMN attenuates the excessive proliferation of PASMCs induced by $M C T$. Immunohistological staining was used to examine the expression of $\alpha$-SMA in rat lung tissues. The results revealed that $\alpha$-SMA was significantly upregulated in MCT-treated rats relative to the control group, but was inhibited following high-dose FMN treatment $(\mathrm{P}<0.01$; Fig. 4A and C). The proliferative ability of PASMCs was analyzed by immunostaining and western blot analysis for PCNA. There was a significant increase in PCNA-positive cells following MCT treatment; however, high-dose FMN treatment FMN significantly abrogated this effect $(\mathrm{P}<0.01$; Fig. 4B and D). In addition, the results of western blot analysis for PCNA were consistent with the observations from immunobiological staining $(\mathrm{P}<0.05$; Fig. $4 \mathrm{~B}$ and $\mathrm{E})$.

FMN inhibits the apoptotic resistance of PASMCs induced by MCT. To evaluate the effects of FMN on apoptosis in rats 

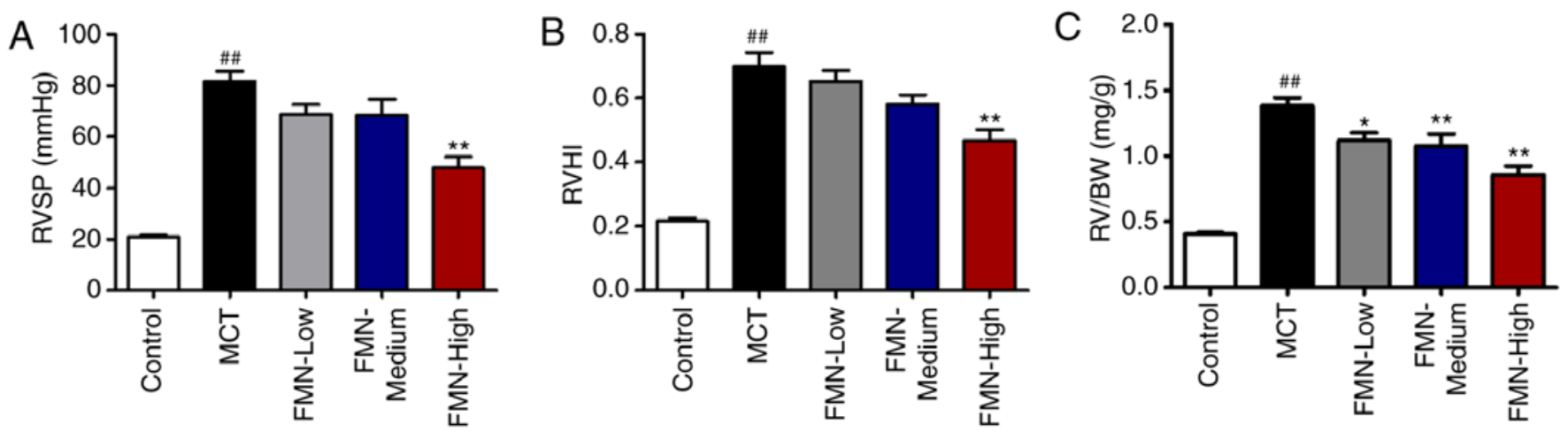

Figure 2. Effects of FMN on hemodynamic alterations and right ventricular hypertrophy. Effect of FMN on (A) RVSP, (B) RVHI and (C) the RV/BW ratio. ${ }^{\# \#} \mathrm{P}<0.01$ vs. the control group. ${ }^{*} \mathrm{P}<0.05,{ }^{* *} \mathrm{P}<0.01$ vs. the MCT group. $\mathrm{n}=8-13$ per group. FMN, formononetin; MCT, monocrotaline; FMN-Low, 10 mg/kg FMN; FMN-Medium, 30 mg/kg FMN; FMN-High, 60 mg/kg FMN; RVSP, right ventricular systolic pressure; RVHI, right ventricular hypertrophy index; RV/BW, right ventricle/body weight ratio.
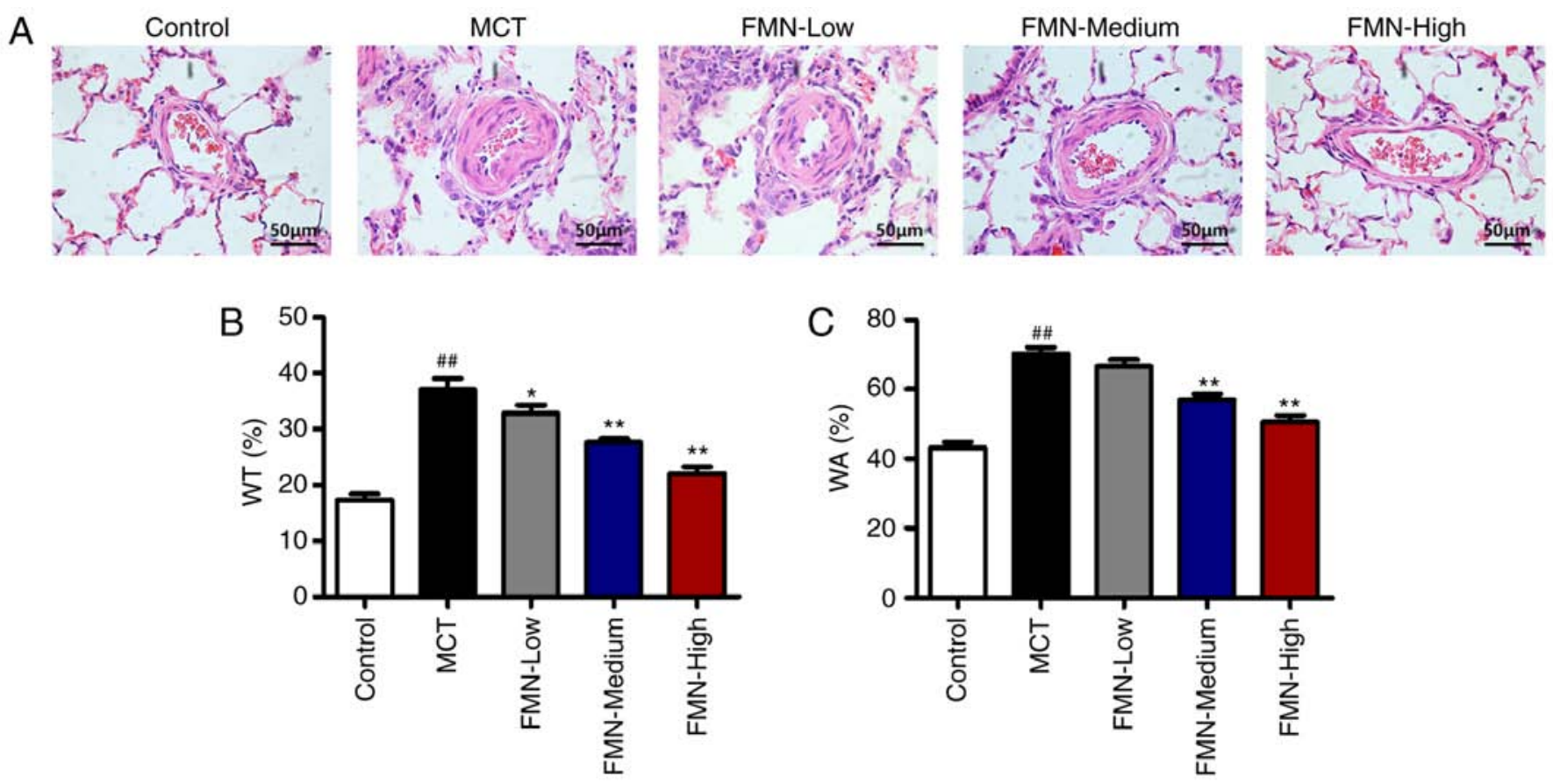

Figure 3. Effects of FMN on pulmonary vascular remodeling. (A) Representative image of pulmonary vascular remodeling as detected by H\&E staining in the lung tissues of rats. Magnification, $\mathrm{x} 400$, scale bar, $50 \mu \mathrm{m}$. Quantification analysis of (B) the WT and (C) WA of the pulmonary arterioles. ${ }^{\# \#} \mathrm{P}<0.01$ vs. the control group. ${ }^{*} \mathrm{P}<0.05,{ }^{* *} \mathrm{P}<0.01$ vs. MCT group. $\mathrm{n}=6$ per group. FMN, formononetin; MCT, monocrotaline; FMN-Low, 10 mg/kg FMN; FMN-Medium, $30 \mathrm{mg} / \mathrm{kg}$ FMN; FMN-High, $60 \mathrm{mg} / \mathrm{kg}$ FMN; WT, vascular wall thickness; WA, vascular wall area.

with PAH, PASMC apoptosis was examined by a TUNEL assay. MCT injection induced a significant decrease in TUNEL-positive cells compared with the control; however, this was reduced following treatment with FMN $(\mathrm{P}<0.01$; Fig. 5A and B). Then, we detected the expression of several apoptosis-associated markers in lung tissues. The expression of Bax and cleaved caspase- 3 in lung tissues were significantly downregulated, while Bcl-2 was upregulated in the MCT group compared with the control. High-dose FMN treatment attenuated decreases in Bax and cleaved caspase-3 expression, and the increase in Bcl-2 expression induced by $\mathrm{MCT}(\mathrm{P}<0.05$; Fig. 5C-E, $\mathrm{G}$ and $\mathrm{H}$ ). In addition, the relative $\mathrm{Bax} / \mathrm{Bcl}-2$ ratio was significantly decreased by MCT compared with the control, but increased following high-dose FMN treatment $(\mathrm{P}<0.05$; Fig. 5F).
FMN inhibits the activation of AKT and ERK induced by MCT. To further explore the effects of FMN on the regulation of the PI3K/AKT signal pathway, we examined the expression of AKT, which serves a crucial role in the PI3K/AKT signaling pathway. It was demonstrated that MCT significantly increased the levels of P-AKT phosphorylation of AKT in lung tissues compared with the control $(\mathrm{P}<0.01$; Fig. $6 \mathrm{~A})$. The increased phosphorylation of AKT was significantly reduced following high-dose treatment with FMN $(\mathrm{P}<0.01)$. In addition, we determined the expression of ERK, which also serves a pivotal role in the pathology of hypoxia and MCT-induced PAH $(28,29)$. The results of the present study revealed that the expression of P-ERK was significantly promoted in the MCT group compared with the control; P-ERK was significantly downregulated following high-dose FMN treatment $(\mathrm{P}<0.05$; Fig. 6B). 
A

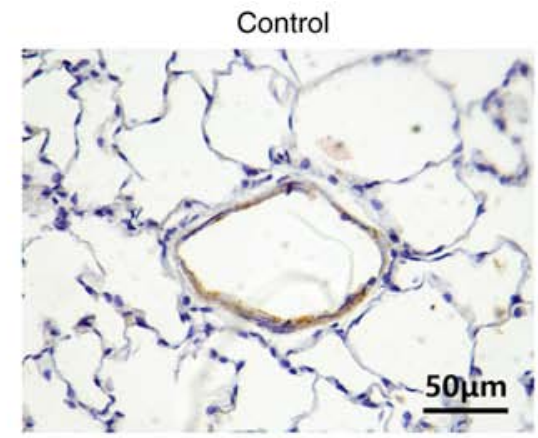

B

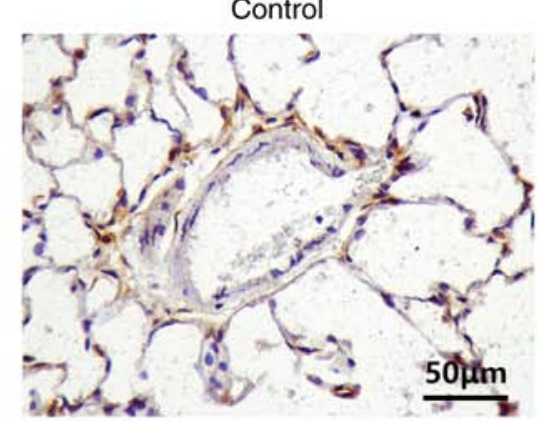

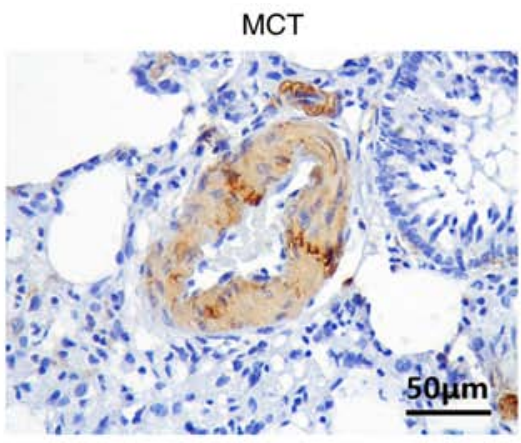

MCT

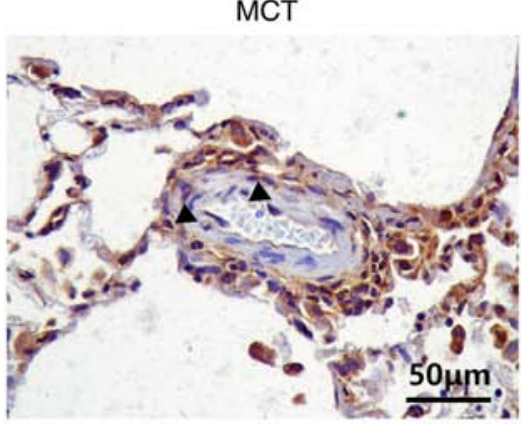

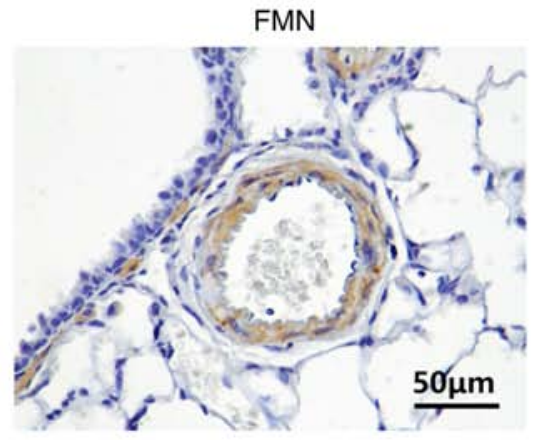

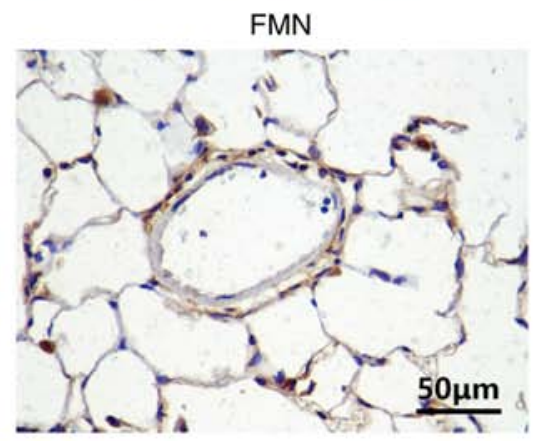

$\mathrm{E}$
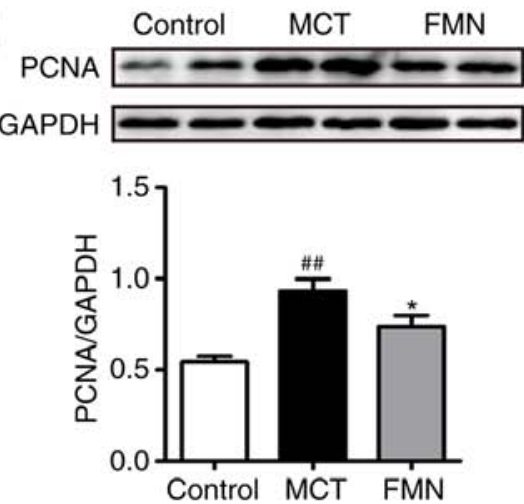

Figure 4. Effects of FMN on the proliferation of PASMCs. Representative images of serial lung sections were analyzed for (A) $\alpha$-SMA and (B) PCNA. Magnification, $\mathrm{x} 400$, scale bar, $50 \mu \mathrm{m}$. The black arrows in the images indicate positive cells. (C) Optical density of $\alpha$-SMA and (D) the percentage of PCNA-positive cells were determined. (E) The expression of PCNA in the lung tissues was examined by western blot analysis. ${ }^{\# \#} \mathrm{P}<0.01$ vs. the control group, ${ }^{*} \mathrm{P}<0.05,{ }^{* *} \mathrm{P}<0.01$ vs. the MCT group. $\mathrm{n}=6$ per group. $\alpha$-SMA, alpha-smooth muscle actin; FMN, $60 \mathrm{mg} / \mathrm{kg}$ formononetin; MCT, monocrotaline; PCNA, proliferating cell nuclear antigen.

\section{Discussion}

PAH is characterized by pulmonary vascular remodeling, leading to the narrowing or occlusion of pulmonary vessels, resulting in elevated pulmonary vascular resistance. It is well reported that pulmonary vascular remodeling is primarily due to the uncontrolled growth and apoptosis resistance of PASMCs (5-7). Therefore, effective therapeutic strategies for treating pulmonary vascular remodeling should be developed. FMN, a major component of isoflavones, exerts a variety of pharmacological effects, which have been associated with the prevention of cardiovascular diseases $(17,18)$. In addition, accumulating evidence suggests that FMN inhibits the proliferation and promotes the apoptosis of tumor cells to exert an antitumor effect. Data from Liang et al (30) revealed that treatment with $50 \mathrm{mg} / \mathrm{kg}$ FMN mediated neuroprotection against cerebral ischemia/reperfusion in rats. For improved efficacy, we decided to increase the dose to $60 \mathrm{mg} / \mathrm{kg}$; following preliminary experiments, we reported that $60 \mathrm{mg} / \mathrm{kg}$ FMN treatment could effectively improve PAH in rats. Therefore, $60 \mathrm{mg} / \mathrm{kg}$ was selected as the treatment dose in the present study. Furthermore, we employed two additional treatment groups, 10 and $30 \mathrm{mg} / \mathrm{kg}$ FMN, to observe whether the effects of FMN on PAH in rats occur in a dose-dependent manner. The results of the present study demonstrated that FMN exerted protective effects on MCT-induced PAH in rats and participated in the regulation of pulmonary vascular remodeling, and PASMC proliferation and apoptosis. Additionally, it was proposed that inhibiting PI3K/AKT and ERK pathways may be involved in mediating the effects of FMN on MCT-induced PAH.

Previous studies found that rats developed severe PAH with high mortality after treatment with MCT $(14,31)$. Furthermore, providing that abnormal hemodynamic changes and right ventricular hypertrophy are representative symptoms of PAH (32-35), long-term survival, RVSP and right ventricular hypertrophy in rats induced by MCT were examined in the 

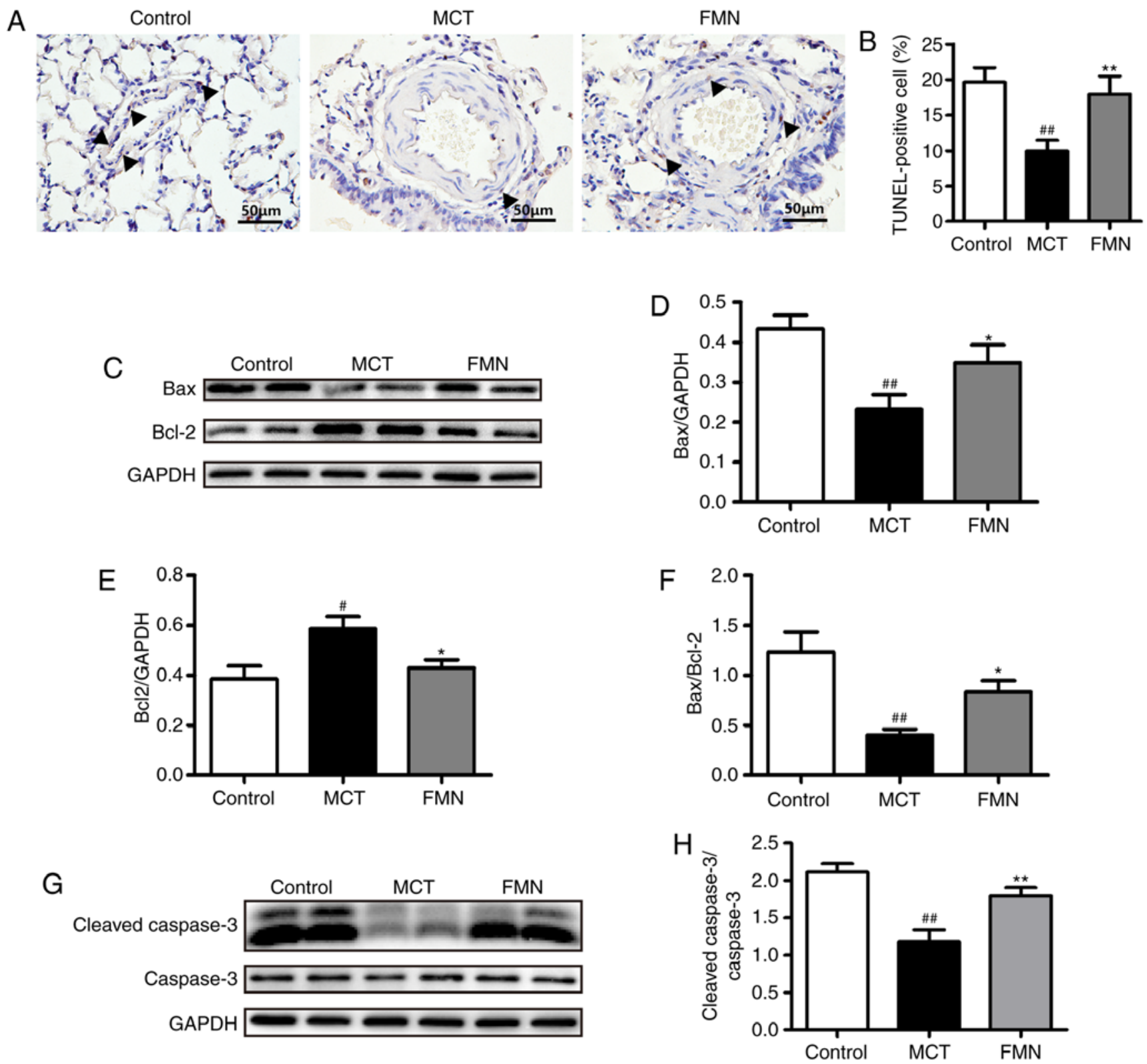

Figure 5. Effects of FMN on apoptosis of PASMCs. (A) Representative images of serial lung sections were analyzed by a TUNEL assay. Magnification, x400, scale bars, $50 \mu \mathrm{m}$. The black arrows indicate positive cells. (B) Percentage of TUNEL-positive cells. The expression of (C and D) Bax, (C and E) Bcl-2 and $\left(\mathrm{G}\right.$ and $\mathrm{H}$ ) cleaved caspase-3 in lung tissues was analyzed by western blotting. (F) The relative expression levels of Bax and Bcl-2 were evaluated. ${ }^{\#} \mathrm{P}<0.05$, ${ }^{\# \#} \mathrm{P}<0.01$ vs. the control group, ${ }^{*} \mathrm{P}<0.05,{ }^{* *} \mathrm{P}<0.01$ vs. the MCT group. $\mathrm{n}=6$. per group. Bcl-2, B-cell lymphoma 2; Bcl-2-associated X protein; FMN, $60 \mathrm{mg} / \mathrm{kg}$ formononetin; MCT, monocrotaline; TUNEL, terminal deoxynucleotidyl-transferase-mediated dUTP nick end.

present study. Our findings revealed that RVSP and RVHI in the MCT group were significantly elevated, and were associated with a high mortality rate. This indicated that the rat model of MCT-induced PAH was successfully established. Furthermore, high-dose FMN treatment significantly improved these adverse phenomena, suggesting the protective effects of PAH.

Several studies reported that the progressive thickening of the pulmonary vascular wall contributes to the development of PAH $(36,37)$. Additionally, FMN was observed to normalize the pulmonary vascular morphology following the induction of PAH via H\&E staining of rat lung tissues in the present study. FMN treatment decreased WT and WA in a dose-dependent manner. Collectively, these findings indicate that FMN inhibited pulmonary vascular remodeling as a protective mechanism against PAH, at least in the rat model of MCT-induced PAH. To further elucidate the possible mechanisms underlying the protective effects on pulmonary vascular remodeling, we selected the high-dose treatment $(60 \mathrm{mg} / \mathrm{kg})$ for subsequent analyses.

FMN has been reported to exert anticancer effects by inhibiting the proliferation and promoting the apoptosis of tumor cells, suggesting that FMN may, by regulating the proliferation and apoptosis of PASMCs, exert protective effects against PAH. The key pathological alteration responsible for increased pulmonary vascular resistance is pulmonary arteriole remodeling, which is primarily attributed to the excessive proliferation of PASMCs (38). In the present study, we detected the expression of $\alpha$-SMA (as a marker of PASMCs) in rat lung tissues. The results revealed that the MCT-induced expression of $\alpha$-SMA was 
A
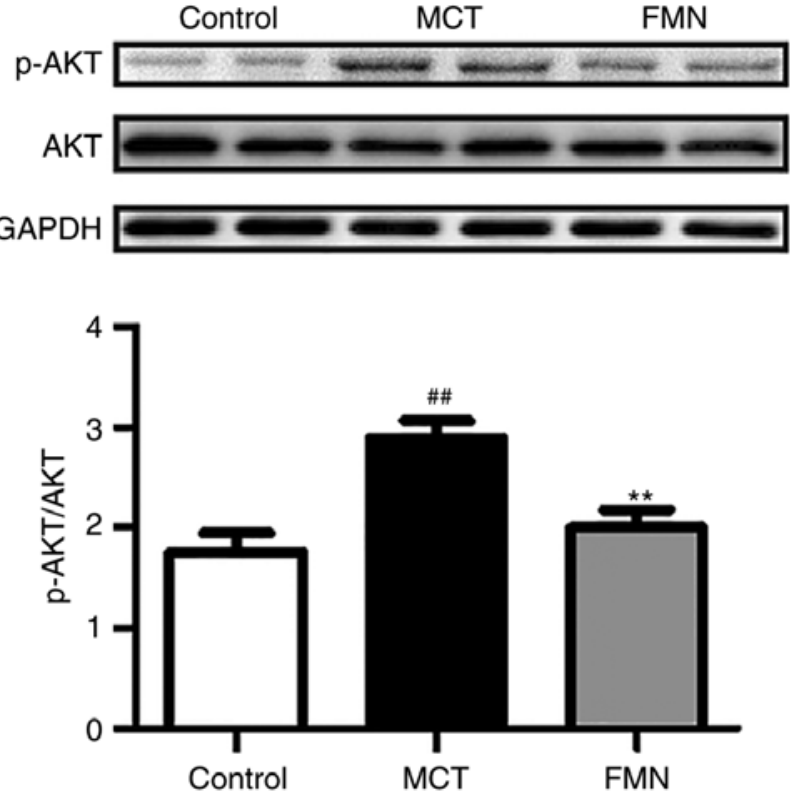

B
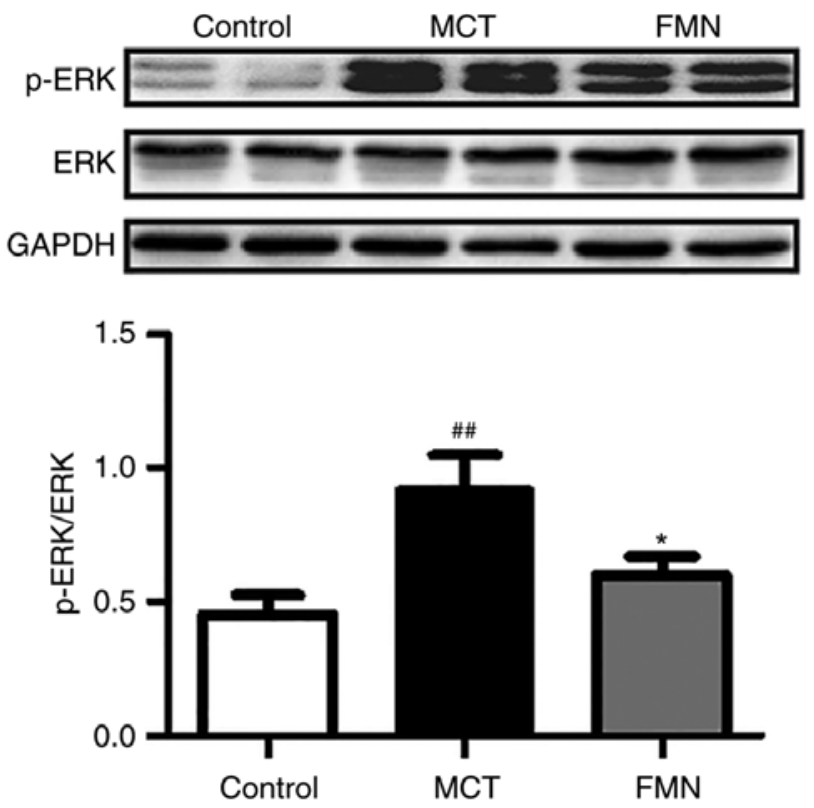

Figure 6. Effect of FMN on the activation of AKT and ERK. (A and B) The expression AKT, P-AKT, ERK and P-ERK in lung tissues was analyzed by western blot analysis. ${ }^{\# \#} \mathrm{P}<0.01$ vs. the control group, ${ }^{*} \mathrm{P}<0.05,{ }^{* *} \mathrm{P}<0.01$ vs. the MCT group. $\mathrm{n}=6$ per group FMN in the figure refers to the high dose $(60 \mathrm{mg} / \mathrm{kg})$ treatment group. AKT, protein kinase B; ERK, extracellular signal-regulated kinase; FMN, $60 \mathrm{mg} / \mathrm{kg}$ formononetin; MCT, monocrotaline; P, phosphorylated.

suppressed by FMN. Immunostaining and western blot analyses of PCNA expression also suggested that FMN reduced the proliferation of PASMCs induced by MCT. In addition, studies in related fields have demonstrated that the pro-apoptotic factor Bax and activated caspase-3 execute the apoptotic program, while $\mathrm{Bcl}-2$, an inhibitor of apoptosis, suppresses the activation of Bax, thereby inhibiting apoptosis $(25,39)$. Under normal circumstances, there is a balance between Bax and Bcl-2, which is dysregulated when pulmonary artery remodeling occurs (40). Multiple studies into the effects of FMN on tumors have revealed that the expression of Bax and cleaved caspase- 3 was downregulated, while that of Bcl-2 was increased (20-23). As we expected, TUNEL staining and western blot analysis of apoptosis-related factors suggested that FMN reversed the suppression of apoptosis induced by MCT. The findings of the present study indicated that FMN ameliorated pulmonary vascular remodeling by regulating the proliferation and apoptosis of PASMCs.

Like many cardiovascular diseases, the development of PAH is closely associated with the aberrant transduction of the PI3K/AKT and ERK signaling pathways (10). Activation of the PI3K/AKT signaling pathway contributes to pulmonary vascular medial thickening, and the proliferation and apoptosis of PASMCs under hypoxic conditions or in rats with MCT-induced PAH (11). AKT serves a major role in the PI3K/AKT signaling pathway, and its activation favors cell survival by regulating apoptotic proteins, including caspases and the Bcl-2 family of proteins (41). ERK is a major factor of the MAPK family, and its activity is considerably increased in various animal models of PAH (37). In addition, decreased ERK activation leads to caspase-3-dependent apoptosis (42). It has been reported that FMN treatment leads to the significant inactivation of AKT and ERK, followed by the upregulation of Bax and downregulation of Bcl-2, which eventually increases the expression of caspase-3 (25). Consistent with previous reports, our results indicate that FMN induced PASMC

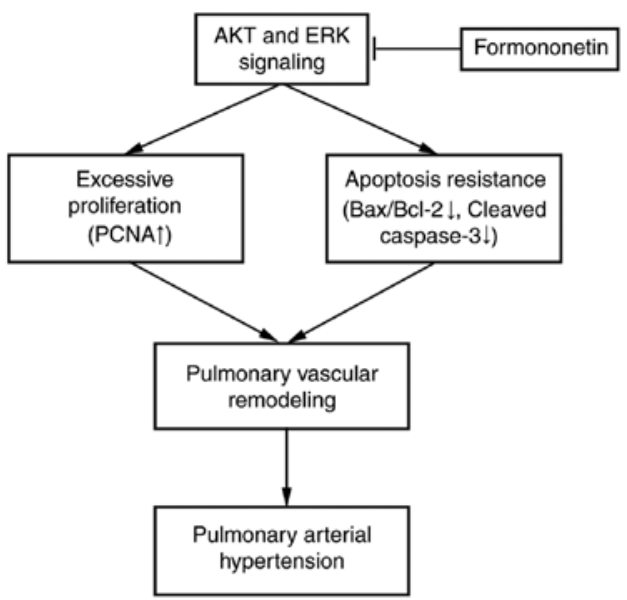

Figure 7. Schematic representation of the mechanism underlying the development of pulmonary arterial hypertension. Straight arrows indicate activation. T-shaped lines indicate inhibition. AKT, protein kinase B; Bcl-2, B-cell lymphoma 2; Bcl-2-associated X protein; ERK, extracellular signal-related kinase; PCNA, proliferating cell nuclear antigen.

apoptosis and inhibits its proliferation, possibly by inactivating the AKT and ERK signaling pathways (Fig. 7).

As previously reported, FMN promotes the apoptosis of tumor cells (21-23). After observing the lung tissue sections of rats by TUNEL staining, we revealed that FMN could promote the apoptosis of PASMCs in vivo. In order to further elucidate the mechanism of FMN pro-apoptosis in vitro, relevant cell experiments must be conducted in the future.

In conclusion, FMN was observed to exert a significant protective effect against MCT-induced PAH and pulmonary vascular remodeling, partially via inhibiting the activation of the PI3K/AKT and ERK pathways. The findings of the present study may provide a theoretical basis for the clinical treatment of PAH. 


\section{Acknowledgements}

Not applicable.

\section{Funding}

No funding was received.

\section{Availability of data and materials}

All data generated or analyzed during the present study are included in this published article.

\section{Authors' contributions}

CC participated in the design of this study and wrote the manuscript. YX, YW and NZ were responsible for data acquisition and statistical analysis. HZ, JX and WL performed the experiments and provided technological assistance. $\mathrm{CZ}$ assisted with data analysis and approved the final version of the manuscript. All authors have read and approved the content of manuscript.

\section{Ethics approval and consent to participate}

The present study was approved by the Ethics Review of Animal Use Application of The Fifth Affiliated Hospital of Wenzhou Medical University (Wenzhou, China).

\section{Patient consent for publication}

Not applicable.

\section{Competing interests}

The authors declare that they have no competing interests.

\section{References}

1. Maron BA and Leopold JA: Emerging concepts in the molecular basis of pulmonary arterial hypertension: Part II: Neurohormonal signaling contributes to the pulmonary vascular and right ventricular pathophenotype of pulmonary arterial hypertension. Circulation 131: 2079-2091, 2015.

2. Simonneau G, Gatzoulis MA, Adatia I, Celermajer D, Denton C, Ghofrani A, Gomez Sanchez MA, Krishna KumarR,Landzberg M, Machado RF, et al: Updated clinical classification of pulmonary hypertension. J Am Coll Cardiol 62 (25 Suppl): D34-D41, 2013.

3. Lai YC, Potoka KC, Champion HC, Mora AL and Gladwin MT: Pulmonary arterial hypertension: The clinical syndrome. Cir Res 115: 115-130, 2014.

4. Guignabert C, Tu L, Girerd B, Ricard N, Huertas A, Montani D and Humbert M: New molecular targets of pulmonary vascular remodeling in pulmonary arterial hypertension: Importance of endothelial communication. Chest 147: 529-537, 2015.

5. Schermuly RT, Ghofrani HA, Wilkins MR and Grimminger F: Mechanisms of disease: Pulmonary arterial hypertension. Nat Rev Cardiol 8: 443-455, 2011.

6. Sakao S and Tatsumi K: Vascular remodeling in pulmonary arterial hypertension: Multiple cancer-like pathways and possible treatment modalities. Int J Cardiol 147: 4-12, 2011.

7. Dumas de la Roque E, Savineau JP, and Bonnet S Dehydroepiandrosterone: A new treatment for vascular remodeling diseases including pulmonary arterial hypertension. Pharmacol Ther 126: 186-199, 2010.

8. Michelakis ED: Pulmonary arterial hypertension: Yesterday, today, tomorrow. Circ Res 115: 109-114, 2014.
9. Montani D, Chaumais MC, Guignabert C, Günther S, Girerd B Jaïs X, Algalarrondo V, Price LC, Savale L, Sitbon O, et al: Targeted therapies in pulmonary arterial hypertension. Pharmacol Ther 141: 172-191, 2014.

10. Archer SL, Weir EK, and Wilkins MR: Basic science of pulmonary arterial hypertension for clinicians: New concepts and experimental therapies. Circulation 121: 2045-2066, 2010.

11. Zhang Q, Cao Y, Luo Q, Wang P, Shi P, Song C, E M, Ren J, Fu B and Sun $H$ : The transient receptor potential vanilloid-3 regulates hypoxia-mediated pulmonary artery smooth muscle cells proliferation via PI3K/AKT signaling pathway. Cell Prolif 51: e12436, 2018.

12. Wu J, Yu Z and Su D: BMP4 protects rat pulmonary arterial smooth muscle cells from apoptosis by PI3K/AKT/Smad1/5/8 signaling. Int J Mol Sci 15: 13738-13754, 2014.

13. Wang W, Prince CZ, Mou Y and Pollman MJ: Notch3 signaling in vascular smooth muscle cells induces c-FLIP expression via ERK/MAPK activation. J Biol Chem 277: 21723-21729, 2002.

14. Wu F, Hao Y, Yang J, Yao W, Xu Y, Yan L, Niu Y, Sun T, Yu J and Zhou R: Protective effects of aloperine on monocrotaline-induced pulmonary hypertension in rats. Biomed Pharmacother 89: 632-641, 2017.

15. Krenn L and Paper DH: Inhibition of angiogenesis and inflammation by an extract of red clover (Trifolium pratense L.). Phytomedicine 16: 1083-1088, 2009.

16. Mu H, Bai YH, Wang ST, Zhu ZM and Zhang YW: Research on antioxidant effects and estrogenic effect of formononetin from Trifolium pratense (red clover). Phytomedicine 16: 314-319, 2009.

17. Zhang S, Tang X, Tian J, Li C, Zhang G, Jiang W and Zhang Z: Cardioprotective effect of sulphonated formononetin on acute myocardial infarction in rats. Basic Clin Pharmacol Toxicol 108: 390-395, 2011.

18. Sun T, Wang J, Huang LH and Cao YX: Antihypertensive effect of formononetin through regulating the expressions of eNOS, 5 -HT2A/1B receptors and $\alpha 1$-adrenoceptors in spontaneously rat arteries. Eur J Pharmacol 699: 241-249, 2013.

19. Li T, Zhao X, Mo Z, Huang W, Yan H, Ling Z and Ye Y: Formononetin promotes cell cycle arrest via downregulation of Akt/Cyclin D1/CDK4 in human prostate cancer cells. Cell Physiol Biochem 34: 1351-1358, 2014.

20. Qi C, Xie M, Liang J, Li H, Li Z, Shi S, Yang X, Wang Z, Tang J and Tang A: Formononetin targets the MAPK and PI3K/AKT pathways to induce apoptosis in human nasopharyngeal carcinoma cells in vitro and in vivo. Int J Clin Exp Med 9: 1180-1189, 2016.

21. Jin YM, Xu TM, Zhao YH, Wang YC and Cui MH: In vitro and in vivo anti-cancer activity of formononetin on human cervical cancer cell line HeLa. Tumor Biol 35: 2279-2284, 2014.

22. Zhang X, Bi L, Ye Y and Chen J: Formononetin induces apoptosis in PC-3 prostate cancer cells through enhancing the Bax/Bcl-2 ratios and regulating the p38/Akt pathway. Nutr Cancer 66: 656-661, 2014

23. Yang Y, Zhao Y, Ai X, Cheng B and Lu S: Formononetin suppresses the proliferation of human non-small cell lung cancer through induction of cell cycle arrest and apoptosis. Int J Clin Exp Pathol 7: 8453-8461, 2014

24. Chen Z, Liu S, Cai Y, Xie K, Zhang W, Dong L, Liu Y, Zheng F, Dun Y and Li N: Suppressive effect of formononetin on platelet-derived growth factor-BB-stimulated proliferation and migration of vascular smooth muscle cells. Exp Ther Med 12: 1901-1907, 2016.

25. Liu Y, He J, Chen X, Li J, Shen M, Yu W, Yang Y and Xiao Z: The proapoptotic effect of formononetin in human osteosarcoma cells: Involvement of inactivation of ERK and Akt pathways. Cell Physiol Biochem 34: 637-645, 2014.

26. Isoda K, Akita K, Kitamura K, Sato-Okabayashi Y, Kadoguchi T, Isobe S, Ohtomo F, Sano M, Shimada K, Iwakura Y and Daida H: Inhibition of interleukin-1 suppresses angiotensin II-induced aortic inflammation and aneurysm formation. Int J Cardiol 270: 221-227, 2018.

27. Zhu N, Zhao X, Xiang Y, Ye S, Huang J, Hu W, Lv L and Zeng C: Thymoquinone attenuates monocrotaline-induced pulmonary artery hypertension via inhibiting pulmonary arterial remodeling in rats. Int J Cardiol 221: 587-596, 2016.

28. Huang Z, Liu Z, Luo Q, Zhao Z, Zhao Q, Zheng Y, Xi Q and Tang Y: Glycoprotein 130 inhibitor ameliorates monocrotaline-induced pulmonary hypertension in rats. Can J Cardiol 32: 1356.e1-1356.e10, 2016

29. Xia S, Tai X, Wang Y, An X, Qian G, Dong J, Wang X, Sha B, Wang D, Murthi P,etal: Involvement of Gax gene in hypoxia-induced pulmonary hypertension, proliferation, and apoptosis of arterial smooth muscle cells. Am J Respir Cell Mol Biol 44: 66-73, 2011. 
30. Liang K, Ye Y, Wang Y, Zhang J and Li C: Formononetin mediates neuroprotection against cerebral ischemia/reperfusion in rats via downregulation of the $\mathrm{Bax} / \mathrm{Bcl}-2$ ratio and upregulation PI3K/Akt signaling pathway. J Neurol Sci 344: 100-104, 2014.

31. Li XL, Guan RJ and Li JJ: Attenuation of monocrotaline-induced pulmonary arterial hypertension in rats by rosuvastatin. J Cardiovasc Pharmacol 60: 219-226, 2012.

32. Wilcox SR, Kabrhel C and Channick RN: Pulmonary hypertension and right ventricular failure in emergency medicine. Ann Emerg Med 66: 619-628, 2015.

33. Tang B, Chen GX, Liang MY, Yao JP and Wu ZK: Ellagic acid prevents monocrotaline-induced pulmonary artery hypertension via inhibiting NLRP3 inflammasome activation in rats. Int $\mathrm{J}$ Cardiol 180: 134-141, 2015

34. Thenappan T, Ormiston ML, Ryan JJ and Archer SL: Pulmonary arterial hypertension: Pathogenesis and clinical management. BMJ 360, j5492, 2018.

35. Ryan JJ and Archer SL: The right ventricle in pulmonary arterial hypertension: Disorders of metabolism, angiogenesis and adrenergic signaling in right ventricular failure. Circ Res 115: 176-188, 2014.

36. Guignabert C, Tu L, Le Hiress M, Ricard N, Sattler C, Seferian A Huertas A, Humbert M and Montani D: Pathogenesis of pulmonary arterial hypertension: Lessons from cancer. Eur Respir Rev 22: 543-551, 2013.

37. Rabinovitch M: Molecular pathogenesis of pulmonary arterial hypertension. J Clin Invest 122: 4306-4313, 2012.
38. Falcetti E, Hall SM, Phillips PG, Patel J, Morrell NW, Haworth SG and Clapp LH: Smooth muscle proliferation and role of the prostacyclin (IP) receptor in idiopathic pulmonary arterial hypertension. Am J Respir Crit Care Med 182: $1161-1170,2010$

39. Bishopric NH, Andreka P, Slepak T and Webster KA: Molecular mechanisms of apoptosis in the cardiac myocyte. Curr Opin Pharmacol 1: 141-150, 2001.

40. Huang X, Zou L, Yu X, Chen M, Guo R, Cai H, Yao D, $\mathrm{Xu}$ X, Chen Y, Ding C, et al: Salidroside attenuates chronic hypoxia-induced pulmonary hypertension via adenosine A 2 a receptor related mitochondria-dependent apoptosis pathway. J Mol Cell Cardiol 82: 153-166, 2015.

41. Rahmani M, Aust MM, Attkisson E, Williams DC Jr, Ferreira-Gonzalez A and Grant S: Dual inhibition of Bcl-2 and Bcl-xL strikingly enhances PI3K inhibition-induced apoptosis in human myeloid leukemia cells through a GSK3- and Bim-dependent mechanism. Cancer Res 73: 1340-1351, 2013.

42. Diab S, Kumarasiri M, Yu M, Teo T, Proud C, Milne R and Wang S: MAP kinase-interacting kinases-emerging targets against cancer. Chem Biol 21: 441-452, 2014.

This work is licensed under a Creative Commons Attribution-NonCommercial-NoDerivatives 4.0 International (CC BY-NC-ND 4.0) License. 